\title{
Day Of The Week Effects In Intra-Day Volatility Patterns Of Equity Markets: A Study Of US And European Stock Markets
}

Deniz Ozenbas, (E-mail: OzenbasD@mail.montclair.edu), Montclair State University Zaman Zamanian

\begin{abstract}
The pattern of intra-day stock price volatility is established in the academic literature as having a Ushape, with heightened volatility at the open and at the close compared to the other periods of the trading day. We establish in this study that there are variations in this pattern across different days of the week. More precisely, we see that the intra-day U-shaped pattern is more accentuated when we take into consideration the day of the week. Using intra-day data from the New York Stock Exchange, London Stock Exchange, Deutsche Boerse and Euronext Paris stock markets we show that Monday openings are consistently more volatile than opening periods of other days, and similarly Friday closings are consistently more volatile than closing periods of other days. These findings indicate the increased difficulty of price discovery just before and after the weekend non-trading period. Variance-ratio statistics are employed to test for the significance of our findings.
\end{abstract}

\section{INTRODUCTION}

$\mathrm{n}$ an ideal world, with informationally efficient markets, prices adjust instantaneously to new information arrival into the market, and volatility is caused solely by the permanent price shifts due to new information. However, trading itself creates price runs and reversals even in the absence of new information and the price discovery process is less efficient than described above. Price changes that are not motivated by new information, but are created by the trading process itself, are generally referred to as trading friction (noise) in academic studies, for example Stoll (2000). These price changes that are due to trading friction are temporary (transient) in nature since they stem from temporary pricing errors. On the other hand, price adjustments that are due to new information are referred to as innovation and are expected to create permanent price shifts.

One of the most important metrics of equity market quality is how well that market is able to accommodate a series of trades and incorporate new information into prices without creating transient price volatility. If the price discovery process is not efficient, then short-term volatility will be heightened in that market. Heightened short-term volatility makes trading more difficult, may scare liquidity away. and can invite more speculative short-term trading into the market. Even though some traders might be able to profit from it, high short-term volatility is costly to the market in aggregate. It discourages trades, lowers portfolio performance for most traders and makes portfolio returns more uncertain for everybody.

The pattern of intra-day stock price volatility is established in the academic literature as having a U-shape, with heightened volatility just after the open and right before the close of trading compared to the other periods of the trading day. The period following the opening and just before the end of trading are periods of particular stress, and require particular attention. These are periods characterized by less efficient price discovery (high trading friction). However, the underlying reasons for the greater uncertainty (and the more difficult price discovery) are different for the opening and closing periods. Price discovery in the morning is more difficult compared to the rest of the trading day mainly because there is information cumulated during the non-trading hours that needs to be translated into prices during this period. Furthermore, following a period of non-trading there could be greater heterogeneity in interpretation of the new information and, thus, more variation in opinions about the direction of stock prices. On the 
other hand, towards the end of the day trading becomes more stressful due to the impending close of trading. Namely, as the market close approaches traders feel a need to close their positions and/or rebalance their portfolios before the non-trading period ends in order to minimize the risk of carrying their positions overnight. This increased trading pressure translates into increased volume and accentuated volatility in stock prices.

We expect that if the above explanations are correct in explaining the intra-day volatility patterns, then we could see even higher levels of stress (and accentuated volatility) during the market open on Mondays and during the market close on Fridays. Since weekends have a two-day long stretch of non-trading in the markets we study, we expect there to be more information accumulation during the weekend period compared to the mid-week overnight period. As a result, the trading periods immediately following the weekend (Monday open) and just before the weekend (Friday close) are expected to be characterized by higher trading friction compared to the mid-week opening and closing periods.

We establish in this study that the intra-day U-shaped pattern is indeed more accentuated when we investigate the periods just after and just before the weekend non-trading periods. Using intra-day data from the New York Stock Exchange, London Stock Exchange, Deutsche Boerse and Euronext Paris we show that Monday openings are consistently more volatile than the opening periods of other days, and similarly Friday closings are consistently more volatile than the closing periods of other days. In other words, we find evidence of day of the week effects (or a weekly seasonality) in intra-day volatility. By investigating this issue across different market centers with different market structures, we establish the universality of this phenomenon, and we are able to draw conclusions on whether or not there is any significant variation across different market structures on this issue.

The remainder of the study is organized as follows. Part 2 reviews the opening procedures in the alternative market centers studied. Part 3 provides the literature review. Part 4 provides an overview of the variance ratio analysis. Part 5 describes the data. Part 6 is a discussion of the methodology and empirical results. Part 7 concludes the study.

\section{OPENING PROCEDURES IN ALTERNATIVE MARKETS}

Opening of trading following a period of non-trading such as the overnight or weekend market close is a particularly stressful period for market participants. It is during this time that all the information that accumulated during the non-trading period needs to be incorporated into stock prices. As a result, during the opening period there is greater uncertainty regarding the stock prices' ability to reflect new information. Furthermore, the variation across market participants in regards to the interpretation of news (also known as heterogeneous expectations) could also add to the stress of this particular period. As a result, market openings are characterized by higher volatility than the rest of the day.

Since the opening interval is an important period of price discovery, many equity markets employ special protocols to facilitate the start of trading. Domowitz and Madhavan (2001) discuss both the importance and the challenges of the opening procedures in an article and argue that "efficient price discovery is a crucial function for securities market. Opening procedures play an especially important role in facilitating price discovery following the enforced trading halt induced by the overnight or weekend non-trading period. Indeed, many markets use special opening procedures designed to provide traders with information regarding market clearing prices with a view toward enhancing liquidity and reducing intra-day price volatility".

Since this study investigates four different equity markets, we can indirectly assess whether or not there are any significant variations in price behavior during the opening across markets. All of the four markets we study are structured differently, and they employ unique protocols to open the market in the morning. This section provides an overview of the different opening procedures followed by the equity markets included in our study.

New York Stock Exchange has a formal opening procedure that may be characterized as an intermediated opening. For each stock on the NYSE, there is a designated specialist whose duty it is to set the opening price. As a 
matter of fact, the specialist has a broader obligation to maintain a fair, competitive, orderly and efficient market and to provide price continuity for the stocks that he or she is responsible for.

Prior to the opening, the specialist observes the overnight accumulation of the limit orders on the limit order book. In addition, market orders are accumulated prior to the open on the Opening Automated Report System (OARS). These orders might come electronically through the SuperDOT system, or they might also come from the floor brokers. Even though the specialists provide indications of the order imbalance to the floor community before the open, they are the only ones who know the individual orders that make up the limit order book and market orders (and hence the order imbalance).

At 9:30, the specialists choose the prices at which the market will open. At this price, all the market on open orders and limit orders (with prices at or better than the opening price) trade. In addition to these obligations and setting the price of the stock, the specialist is also allowed to trade the stock proprietarily, and as a result enjoys, as a trader, the unique informational advantage that stems from being able to observe the order flow. At the open, the specialists, once they set the opening price, must absorb any excess demand or supply at the opening prices from their inventories.

Even though the specialist typically trades at the open either to offset a buy/sell order imbalance or as a proprietary trader, it has been shown that their relative trading volume in larger and more liquid stocks is significantly less than their trading volume in smaller and less liquid stocks. As a result, we expect that the specialist intervention will be less for our NYSE sample that consists of very large capitalization and highly liquid stocks.

In a recent study, Madhavan and Panchapagesan (2000) show that the specialist intervention facilitates the price discovery at the open in the NYSE and makes the opening price more efficient compared to a fully automated opening procedure (i.e. an opening call auction) without specialist intervention. The way the authors define efficiency of the opening price is by comparing that price to the future $3: 00 \mathrm{pm}$ price observed during the same day. The authors argue that this increased efficiency reflects the information specialists gain from observing the evolution of the limit order book and the requirement they have to provide price stabilization. However, needless to say, call auctions can be designed in a variety of ways. The comparison the authors make is between the specialist-intermediated opening at the NYSE and an automated call auction without full transparency that would have taken place if there were no specialist intervention at the NYSE opening. It is not clear, however, whether the benefits of the specialist intervention would have remained for a call auction that provides full transparency before the market open (such as the case with the opening protocol of Euronext Paris).

The European markets we investigate, the Deutsche Boerse, Euronext Paris and London Stock Exchange, are all electronic order-driven markets, and in this respect they are relatively similar to the NYSE. However, none of them has a physical trading floor. Also, the London Stock Exchange comes from a dealer-market structure that used to resemble the Nasdaq Stock Market. All of these European markets open their trading in the morning with a call auction. After the opening auction in each market, trading starts in the form of continuous trading. At the Euronext Paris and Deutsche Bourse the opening call auction takes place at 9:00 am. On the other hand, the opening call auction takes place at 8:00 am on the London Stock Exchange. However, if we take into consideration the one-hour difference between England and the Continental Europe, then we see that the start of trading is synchronized for all three European markets studied.

One of the more important differences between Deutsche Boerse and Euronext Paris with respect to their opening procedures is that Deutsche Borse has intermediated openings (with the help of a "kursmakler"), whereas the opening at the Euronext Paris is fully automated. Deutsche Borse has a specialist system that is similar to the specialist system of the NYSE. Again there is only one specialist in the Deutsche Borse, called "Kursmakler", for each stock, and one kursmakler can make the market for multiple stocks. Furthermore, the kursmakler is also able to trade proprietarily for her account. At the opening call auction, it is the kursmakler who sets the opening price based on the order flow that came in during the non-trading period. 
There are no specialists at Euronext Paris and as a result the opening price that is the outcome of the opening call auction depends solely on the orders that came in during the pre-opening period. The pre-opening period of the Euronext Paris is further characterized by a high degree of transparency, where the traders can observe the evolution of the limit order book. Furthermore, until it is very close to the opening, traders are able with relative ease to cancel the orders they placed. In contrast, the Deutsche Borse call auctions are characterized by a relative lack of transparency.

Biais, Hillion and Spatt (1999) investigate the price formation process during the pre-opening period at Paris Bourse. The authors show that the indicative opening price is very informative in reflecting the overnight information accumulation. Learning among traders that leads to price discovery does take place during the pre-opening period. However, the high level of transparency, coupled with the ease with which traders can cancel their orders before the opening auction might let them "game" the market by withholding their most informative orders until the market is very close to opening (this phenomenon is also known as "suckering"). Pagano and Schwartz (2003) recently show that price discovery at the Euronext Paris' market opening (and close) was improved by the institution of a call auction to close the market in 1996.

London Stock Exchange made a number of enhancements to their opening call auction on May 30th 2000, simultaneously with introducing the closing call auction. These enhancements were the availability of market orders in addition to the limit orders, calculation and dissemination of an indicative auction price during the call period, random end to the auction call period, auction call period extensions under specific situations such as significant price moves, and alignment of the matching algorithm with the other European markets.

London Stock Exchange has a competing dealer market structure (that is distinct from the other markets studied and is similar to the Nasdaq Stock Market) in addition to their electronic SETS system. There is a further aspect that distinguishes London Stock Exchange from the other markets studied. Trading on the LSE is dominated by the institutional traders, in contrast to a more balanced combination of retail and institutional trading observed in the other markets. Clearly, this is an important factor that might impact the trading culture on the LSE not only during the trading hours but also during the period that covers the opening.

\section{LITERATURE REVIEW}

In a comprehensive study that investigates liquidity and trading activity in the NYSE over the period of 1988 to 1998, Chordia, Roll, and Subrahmanyam (2001) find a relative lack of change in absolute daily prices (0.56\%) compared to average absolute change in daily liquidity variables (about $2 \%$ ), average absolute change in daily depth variables (about 4-5\%), and average absolute change in daily trading activity variables (15-20\%). In this study, the authors use quoted and effective spreads and depth as the measures of daily liquidity and volume and number of daily transactions are used as the measures of trading activity. This relative lack of change in absolute daily prices is a surprising result given the high levels of intra-day volatility documented in the current study and elsewhere, and suggests that there are big gyrations during the trading day that are not manifest in longer periods, even on a daily basis. It has been suggested in the academic literature that the day of the week might have an effect on liquidity and trading. In fact, Chordia et al find that market is more liquid and trading activity is higher on Tuesdays through Thursdays compared to Mondays and Fridays. Foster and Wiswanathan $(1990,1993)$ show that trading volume is low and adverse selection costs are high on Mondays compared to other days. We also investigate the day of the week effect (or a weekly seasonality) in this study.

A sub-field of research in market quality is a transaction level analysis of the period surrounding the opening of trading after a period of non-trading (e. g. the overnight period or a trading halt). Typically these studies find that the opening of trading after a period of non-trading is a stressful period, and that informal or formal mechanisms are necessary to improve the efficiency of the opening. Some of these studies are reviewed in part 2 of the current study, such as Biais, Hillion, Spatt (1999) and Madhavan and Panchapagesan (2000).

Intra-day patterns in volatility, and volume were first documented by Wood, McInish and Ord (1985), Jain and Joh (1988) and Harris (1986). Subsequently, this area has been investigated by other academic studies including 
Madhavan et al (1997) and Ozenbas (2006). Generally, these studies find a U-shape in volume and volatility across the markets studied. Admati and Pfleiderer (1988) provide a theoretical explanation for the intra-day U-shaped patterns in volume and volatility in which concentrated-trading patterns arise endogenously as a result of the strategic behavior of liquidity traders and informed traders. Several other researchers argue that the trading costs should increase towards the end of the trading day, creating more volume and volatility near the closing of trading. Examples include Bessembinder (1994), and Brock and Kleidon (1992).

One of the more important differences between the above studies and the current one is that, in the current study the patterns of intra-day volatility is investigated separately for each of the five days of the week and contrasted across days in order to find the day of the week effects in these intra-day patterns. In the studies mentioned above, either intra-day patterns are investigated without differentiating between the days of the week, or the day of the week effects are investigated without differentiating between the time during the trading day (i.e. the opening versus the closing period of the trading day). In the current study we attempt to consolidate the two dimensions of inter-temporal investigation: the intra-day patterns of equity price volatility according to both the time of the day and the day of the week

\section{A BRIEF DISCUSSION OF VARIANCE RATIOS}

In this study, we use variance ratios to measure the extent of accentuated short-term volatility. An important implication of random walk hypothesis is that variance of the random walk increments (measured by the natural logarithm of price relatives) is a linear function of the time period. In other words, if returns of stock prices follow a random walk, it should not matter whether we use hourly, daily or weekly time increments to measure the return variance. They should all give approximately the same variance once they are adjusted for the differencing interval.

For example, under the random walk hypothesis, $\operatorname{Var}\left(r_{t}(2)\right)$ should be twice $\operatorname{Var}\left(r_{t}(1)\right)$ and the following variance ratio $\operatorname{VR}(2)=\frac{2 \operatorname{Var}\left[\mathrm{r}_{\mathrm{t}}(1)\right]}{\operatorname{Var}\left[\mathrm{r}_{\mathrm{t}}(2)\right]}$ should be one. However, existence of serial autocorrelation in stock returns causes the variance ratios to differ from one. Again we can demonstrate this for the two-period variance ratio as follows:

$$
\begin{aligned}
& \operatorname{VR}(2)=\frac{2 \operatorname{Var}\left[\mathrm{r}_{\mathrm{t}}(1)\right]}{\operatorname{Var}\left[\mathrm{r}_{\mathrm{t}}(2)\right]}=\frac{2 \operatorname{Var}\left[\mathrm{r}_{\mathrm{t}}\right]}{\operatorname{Var}\left[\mathrm{r}_{\mathrm{t}}+\mathrm{r}_{\mathrm{t}-1}\right]} \\
& \operatorname{Var}\left[r_{t}+r_{t-1}\right]=\operatorname{Var}\left[r_{t}\right]+\operatorname{Var}\left[r_{t-1}\right]+2 \operatorname{Cov}\left[r_{t}, r_{t-1}\right]=2 \operatorname{Var}\left[r_{t}\right]+2 \operatorname{Cov}\left[r_{t}, r_{t-1}\right] \\
& \operatorname{VR}(2)=\frac{2 \operatorname{Var}\left[\mathrm{r}_{\mathrm{t}}\right]}{2 \operatorname{Var}\left[\mathrm{r}_{\mathrm{t}}\right]+2 \operatorname{Cov}\left[\mathrm{r}_{\mathrm{t}}, \mathrm{r}_{\mathrm{t}-1}\right]} \\
& \operatorname{VR}(2)=[1+\rho(1)]^{-1}
\end{aligned}
$$

where $\rho(1)$ is the first order autocorrelation of the return series. Since the denominator of the above variance ratio is the variance of two-period returns series, we are only concerned with the first order autocorrelation. However, when the long-period return series are more than double the short-period return series, then we need to investigate higher order autocorrelation terms than the first order. The VR(2) formula derived above shows that while a positive autocorrelation causes the VR(2) to be less than one, negative serial autocorrelation will cause the VR(2) to be greater than one. In other words, under positive autocorrelation, variance of longer periods will be larger than sum of the variances of shorter periods. Under negative autocorrelation, variance of longer periods will be smaller than sum of the variances of shorter periods. Similarly, when you compare two variances calculated using the same differencing 
interval (such as the half-hour differencing intervals used in this study), the variance ratio should not differ from unity either.

\section{DATA}

Four markets are studied: The New York Stock Exchange in the US and the Euronext Paris (Paris Bourse), Deutsche Boerse and the London Stock Exchange in Europe. Intraday trades and quotes data for NYSE were obtained from the TAQ (Trades and Quotes) database. The study period is July-December 2000. We include in our study the stocks that make up a major index for all market centers. For NYSE, we choose the stocks that were a part of the S\&P 100 index on December 31, 2000. Of course, the S\&P 100 index contains a few Nasdaq stocks and we eliminate these stocks from our NYSE sample. In addition, we perform extensive error-filtering of the data.

The three foreign markets that are studied are the Euronext Paris (Paris Bourse), Deutsche Boerse and the London Stock Exchange. For each market we study the transaction records, during the year 2000, of stocks that make up a major index. We use the BDM database of the Paris Bourse for the transactions of the stocks that make up the CAC 40 index, and the Transaction Data Service database of the London Stock Exchange for the transactions of the stocks that make up the FTSE 100 index. The transactions database of the stocks that make up the DAX 30 index was obtained from the Deutsche Boerse.

Trading hours at the Paris Bourse at the beginning of 2000 were 9:00 am to 5:00 pm. The hours were extended on April 1st to 9:00 am to 5:30 pm. The market opens with a call auction within the first minutes after 9:00 am, and there is another call auction that takes place about 5 minutes after the close. We study the period after the rule change that corresponds to the longer trading hours, i.e. the period from April 2nd to December 31st. Trading hours for Deutsche Boerse at the beginning of the year were 9:00 am to 5:30 pm, but similar to Paris Bourse they were extended to 9:00 am to 8:00 pm on June 2nd, 2000. Again similar to the Paris Bourse, trading opens with an opening call auction that takes place within the first few minutes after 9:00 am. There is an intraday call auction a few minutes after 1:00 pm. In addition, there is a call auction that takes place about 5-10 minutes after the close. Before the extension of trading hours, the closing call was around 5:40 pm. After the extended trading hours, this call is kept as the second intraday call auction and the closing call auction takes place few minutes after 8:00 pm. Again, we study the period that corresponds to the longer trading hours, which is from June 3rd to December 31st. Trading hours for the London Stock Exchange are 8:00 am to 4:30 pm. A closing call auction was introduced on May 30th 2000 to the London Stock Exchange. Starting with this date, trading has been ending with a call auction that takes place several minutes after 4:30 pm. Once again we study the period after the rule change which corresponds to the period with the closing call auction, i.e. from June 1st to December $31^{\text {st }}$. To check the robustness of our results we ran our tests for the beginning half of 2000 for all markets. The results are consistent with the results form study periods reported, and are available upon request.

\section{EMPIRICAL METHODOLOGY AND RESULTS}

In all the markets, our findings confirm a $\mathrm{U}$-shaped intra-day volatility pattern, with a more pronounced spike at the beginning of the trading day. These findings confirm that the period following the opening and just before the end of trading are periods of particular stress, and require particular attention. Intra-day volatility patterns show that volatility is accentuated in all markets studied during the beginning of trading in the morning and towards the close of trading. This result clearly suggests that these are periods characterized by less efficient price discovery and high trading friction. However, as discussed earlier the underlying reasons for the greater uncertainty and the more difficult price discovery are different for the opening and closing periods. Price discovery in the morning is more difficult compared to the rest of the trading day mainly because there is information cumulated during the non-trading hours that needs to be translated into prices during this period. Furthermore, following a period of non-trading there could be greater heterogeneity in interpretation of the new information and, thus, more variation in opinions about the direction of stock prices. On the other hand, towards the end of the day trading becomes more stressful due to the impending close of trading. Namely, as the market close approaches traders feel a need to close their positions and/or rebalance their portfolios before the non-trading period ends in order to minimize the risk of carrying their positions overnight. This increased trading pressure translates into increased volume and accentuated volatility in stock prices. 
We expect that if the above explanations are correct in explaining the intra-day volatility patterns, then we could see even higher levels of stress (and accentuated volatility) during the market open on Mondays and during the market close on Fridays. Since weekends have a two-day long stretch of non-trading in the markets we study, we expect there to be more information accumulation during the weekend period compared to the mid-week overnight period. As a result, the trading periods immediately following the weekend (Monday open) and just before the weekend (Friday close) are expected to be characterized by higher trading friction compared to the mid-week opening and closing periods. In order to test for the day of the week effects, we calculate the intra-day variances for each day of the week separately. We then compare the Monday opening half-hour volatility to the mid-week (Wednesday) opening half-hour volatility and the Friday closing half-hour volatility to the mid-week (Wednesday) closing half-hour volatility. Findings are summarized in Table 1. Results support our expectations since in the majority of markets Monday opening half-hour and Friday closing half-hour variances are more accentuated compared respectively to the opening and closing half-hours of Wednesday. The ratios are statistically higher than unity in most of the cases (but are never statistically significantly lower than unity). Especially for the London Stock Exchange, opening and closing volatility is particularly more accentuated on Mondays and Fridays respectively. This result is consistent with Ozenbas (2006) where it is reported that the London Stock Exchange has the most pronounced U-shaped intra-day pattern of volatility among the same markets studied.

For Deutsche Boerse, we present two sets of findings for the closing period. The reason for this is that even though trading hours were recently extended in this market during our study period, this rule change was relatively unsuccessful with very low trading volume during these extended hours. In other words, the original 5:30 pm still remained the effective close for this market. As a result, we calculate and present two sets of closing results, one for the actual 8:00 pm close and one for the effective 5:30 pm close. We find that the Friday closing call auction at 8:00 $\mathrm{pm}$ in Deutsche Boerse is significantly more volatile compared to the mid-week closing call auction at 8:00 pm. This finding suggests that even though there is not much trading during the extended hours, the last call auction before the weekend is an important vehicle of price discovery.

More detailed findings are presented in Figures 1 through 8. We present the levels of the opening half-hour volatility and the closing half-hour volatility for each day of the week in Figures 1 through 4 . In all markets studied the opening half-hour volatility is highest on Mondays, consistent with our expectations. Similarly, in all markets studied the closing half-hour volatility is highest on Fridays, also consistent with our expectations. In agreement with other studies mentioned earlier, the opening half-hour volatility is higher than the closing half-hour volatility for all markets studied. Also, in Figure 4, consistent with the low volume of trading during the extended trading hours in Deutsche Bourse, the absolute volatility of the 8:00 pm close is lower than the absolute volatility of the effective 5:30 pm close for each day of the week.

In Figures 5 through 8, we present the U-shaped intra-day volatility pattern for Mondays contrasted with the U-shaped intra-day volatility pattern for Fridays for each of the four markets. For all the markets, the opening halfhour volatilities on Mondays are higher than the opening half-hour volatilities on Fridays and the reverse is true for the closing half-hour volatilities. This is again in line with our expectations and indicates the increased difficulty of price discovery before and after the weekend non-trading period. On the other hand, we do not find any such clear pattern when we investigate the mid-day periods. We find, for the mid-day periods, relatively comparable intra-day volatilities for Mondays versus Fridays for all of our four markets. Once again, despite the lack of success of the extended trading hours for Deutsche Boerse, Friday closings appear to be a significant vehicle of price discovery for this market judging by the relatively high closing half-hour volatility in Figure 8.

\section{CONCLUSION}

In this study, we investigate the level of short-term volatility accentuation according to the day of the week in the most liquid stocks of four different equity markets: New York Stock Exchange, London Stock Exchange, Deutsche Boerse, and Euronext Paris. In all the markets in this study, we choose the individual stocks that make up a major index during our study period. 
The quality of a market can best be evaluated during stressful periods and market openings and closings certainly fit that description. Furthermore, if that stress is linked to the pressures of trading right before or immediately after the non-trading period, we should see that the opening period of a Monday and the closing period of a Friday are even more volatile compared to the mid-week opening and closing periods. In this study, we study the intra-day patterns of volatility using half-hour differencing intervals and find evidence supporting our hypothesis. Opening halfhour volatility appears to be the highest on Mondays and closing half-hour volatility appears to be the highest on Fridays for all four markets we study with particularly significant results for the London Stock Exchange.

An interesting venue to further this analysis would be to investigate the extent to which the pattern of news releases in each of these markets matters. Similarly, another area that is a natural extension of this study is to find whether there are any variations in the volatility patterns of cross-listed stocks that trade simultaneously in multiple markets with overlapping trading hours. All in all, the short-term analysis of volatility raises many exciting questions that should lead the way to new and fruitful research.

\section{REFERENCES}

1. Admati, R. Anat and Paul Pfleiderer, 1988, A theory of intraday patterns: Volume and price variability, The Review of Financial Studies 1, 3-40.

2. Bessembinder, H. 1994, Bid-Ask Spreads in the Inter-Bank Foreign Exchange Markets, Journal of Financial Economics 35, 317-348.

3. Biais, Bruno, Pierre Hillion, and Chester Spatt, 1999, Price discovery and learning during the pre-opening in the Paris Bourse, Journal of Political Economy 107, 1218-1248.

4. Brock, W. and A. Kleidon, 1992, Periodic Market Closure and Trading Volume: A Model of Intraday Bids and Asks, Journal of Economic Dynamics and Control 16, 451-489.

5. Campbell, J., A. Lo, and C. MacKinlay, 1997, The Econometrics of Financial Markets, Princeton University Press.

6. Cao, Charles, Eric Ghysels, and Frank Hatheway, 2000, Price Discovery without Trading: Evidence from the Nasdaq Preopening, Journal of Finance 56,1339-1365.

7. Chordia Tarun, Richard Roll, and Avanidhar Subrahmanyam, 2001, Market liquidity and trading activity, Journal of Finance 56, 501-530.

8. Domowitz, I. and A. Madhavan, 2001, Open Sesame: Alternative Opening Algorithms in Security Markets, The Electronic Call auction: Market Mechanism and Trading (ed. R.A. Schwartz), Kluwer Academic Publishers.

9. Foster, D. and S. Wiswanathan, 1990, A Theory of Interday Variations in Volumes, Variances, and Trading Costs in Securities Markets, The Review of Financial Studies 3, 593-624.

10. Foster, D. and S. Wiswanathan, 1993, Variations in Trading Volume, Return Volatility, and Trading Costs: Evidence on Recent Price Formation Models, Journal of Finance 48, 187-211.

11. Harris, Lawrence, 1986, A transaction data study of weekly and intradaily patterns in stock returns, Journal of Financial Economics 16, 99-118.

12. Hasbrouck, Joel and Robert A. Schwartz, 1988, Liquidity and execution costs in equity markets, Journal of Portfolio Management Spring, 10-15.

13. Jain, Prem and Gun-Ho Joh, 1988, The Dependence Between Hourly Prices and Trading Volume, Journal of Financial and Quantitative Analysis 23, 269-285.

14. Madhavan, A. and V. Panchapagesan, 2000, Price discovery in auction markets: A look inside the black box, Review of Financial Studies 13, 627-658.

15. Madhavan, A., M. Richardson, and M. Roomans, 1997, Why do security prices change? A transaction-level analysis of NYSE stocks, Review of Financial Studies 10, 1035-1064.

16. Ozenbas, D. 2006, Pattern of Short-Term Volatility Accentuation Within the Trading Day: An Investigation of the US and European Equity Markets, International Business \& Economics Research Journal, forthcoming.

17. Pagano, M.S. and R.A. Schwartz, 2003, A Closing Call's Impact on Market Quality at Euronext Paris. Journal of Financial Economics, Volume 68, pp. 439-484.

18. Stoll and Hans, 2000, Friction, Journal of Finance 55, 1479-1515. 
19. Wood, Robert A., Thomas H. McInish, and Keith Ord, 1985, An investigation of transactions data for NYSE stocks, Journal of Finance 40, 723-741.

\section{Table 1: Day Of The Week Variance Ratios For Opening / Closing Half Hour Periods}

Ratio of opening half hour variance on Monday to the opening half hour variance on Wednesday is presented in Column A. Ratio of closing half hour variance on Friday to the closing half hour variance on Wednesday is presented in Column B.. The study period is July-December 2000 for New York Stock Exchange and Nasdaq Stock Market, June-December 2000 for Deutsche Boerse and London Stock Exchange, and April-December 2000 for Euronext Paris. Deutsche Boerse (1) statistics are calculated using 5:30 pm as the effective closing time, and Deutsche Boerse (2) statistics are calculated using the actual 8:00 pm as the closing time during the second study period.

\begin{tabular}{|c|c|c|}
\hline Second Study Period & $\begin{array}{c}\text { A } \\
\text { Monday Opening HH / Wednesday } \\
\text { Opening HH } \\
\end{array}$ & \begin{tabular}{|c|} 
B \\
Friday Closing HH / Wednesday Closing \\
HH \\
\end{tabular} \\
\hline New York Stock Exchange & 1.017 & $1.139 *$ \\
\hline London Stock Exchange & 1.515 & 1.416 \\
\hline Euronext Paris & $1.221 *$ & $1.155^{*}$ \\
\hline Deutsche Boerse (1) & 1.072 & $1.199 *$ \\
\hline Deutsche Boerse (2) & 1.072 & 1.625 \\
\hline
\end{tabular}

* indicate significantly different than unity at the $10 \%$ confidence level.

Bold entries indicate significantly different than unity at the 5\% confidence level.

Bold and italic entries indicate significantly different than unity at the $1 \%$ confidence level.

Figure 1: Day Of The Week Volatility, New York Stock Exchange

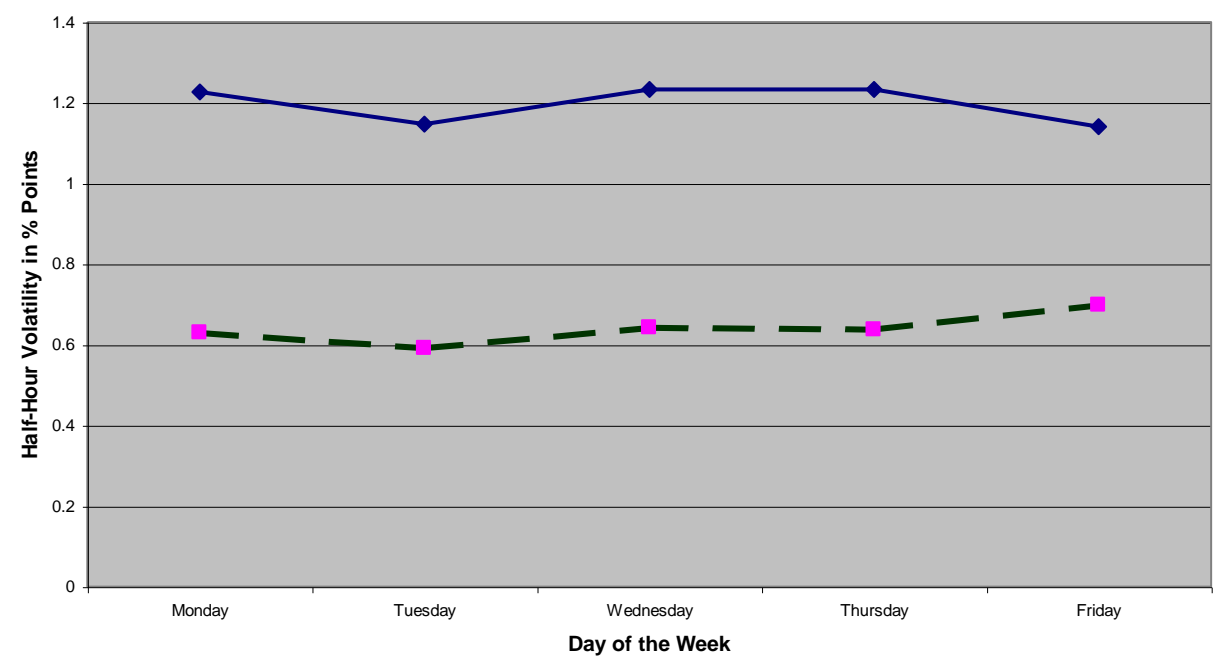

Opening half hour volatility and closing half hour volatility are calculated for each day of the week separately. The unbroken (top) line represents the opening half hour volatility and the broken (bottom) line represents the closing half hour volatility. Opening half-hour period does not contain the over-night price changes. The study is July-December 2000. 
Figure 2: Day Of The Week Volatility, London Stock Exchange

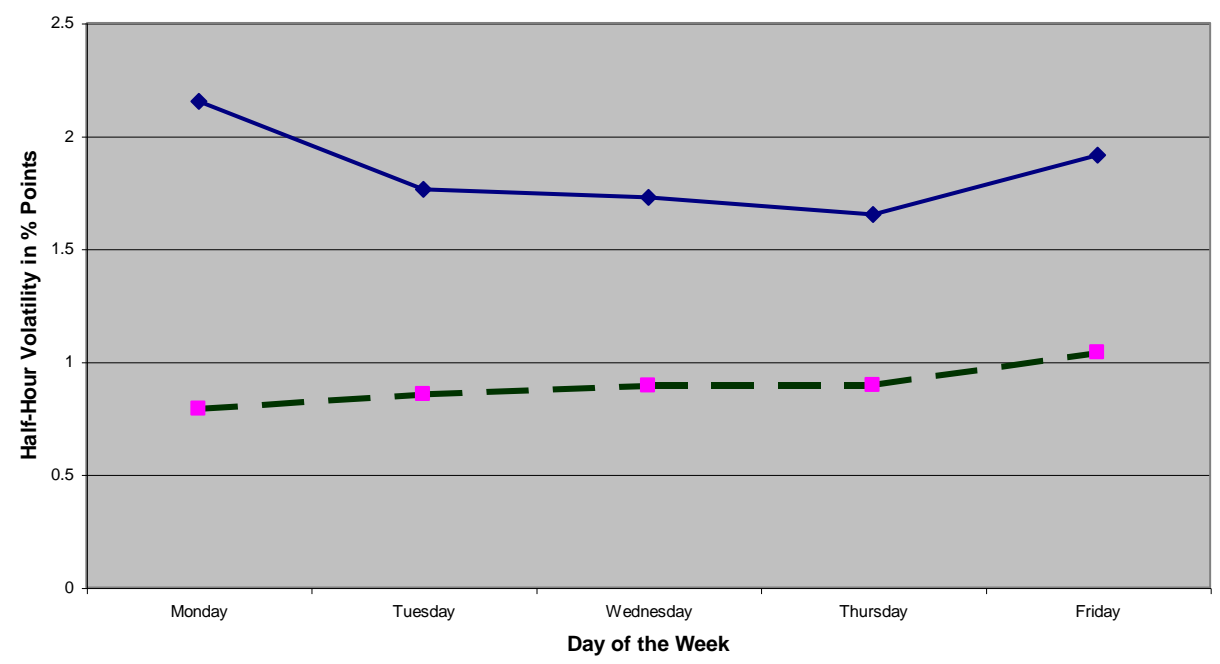

Opening half hour volatility and closing half hour volatility are calculated for each day of the week separately. The unbroken (top) line represents the opening half hour volatility and the broken (bottom) line represents the closing half hour volatility. Opening half-hour period does not contain the over-night price changes. The study period is June-December 2000.

Figure 3: Day Of The Week Volatility, Euronext Paris

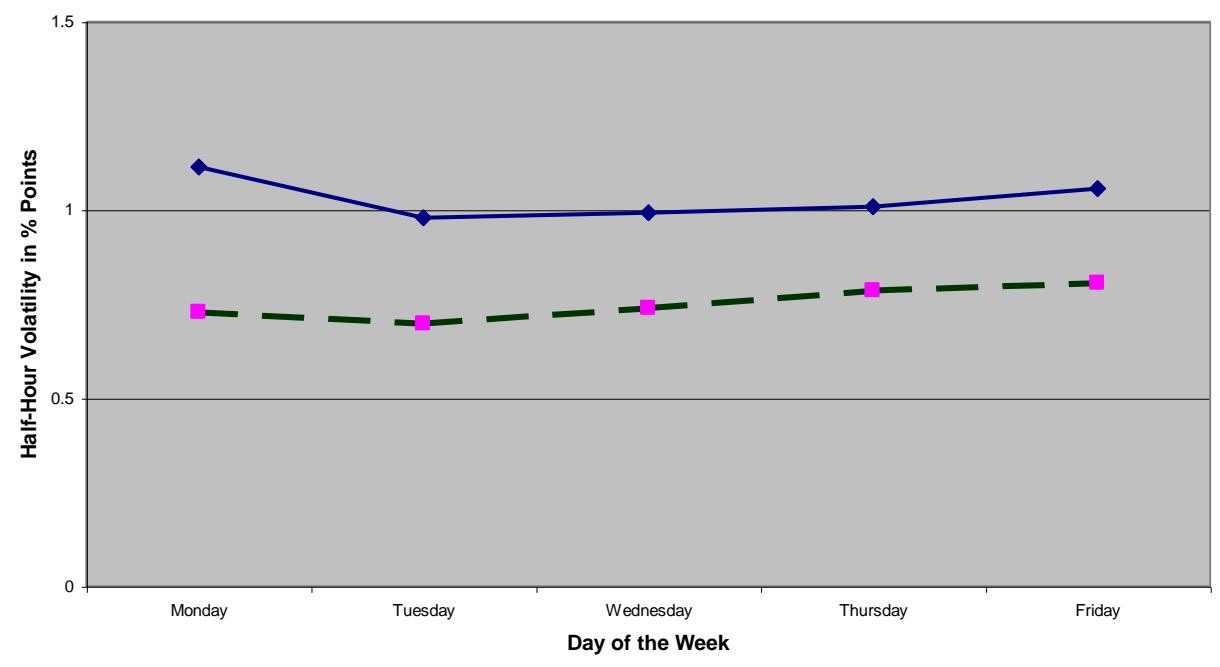

Opening half hour volatility and closing half hour volatility are calculated for each day of the week separately. The unbroken (top) line represents the opening half hour volatility and the broken (bottom) line represents the closing half hour volatility. Opening half-hour period does not contain the over-night price changes. The study period is April-December 2000. 
Figure 4: Day Of The Week Volatility, Deutsche Boerse

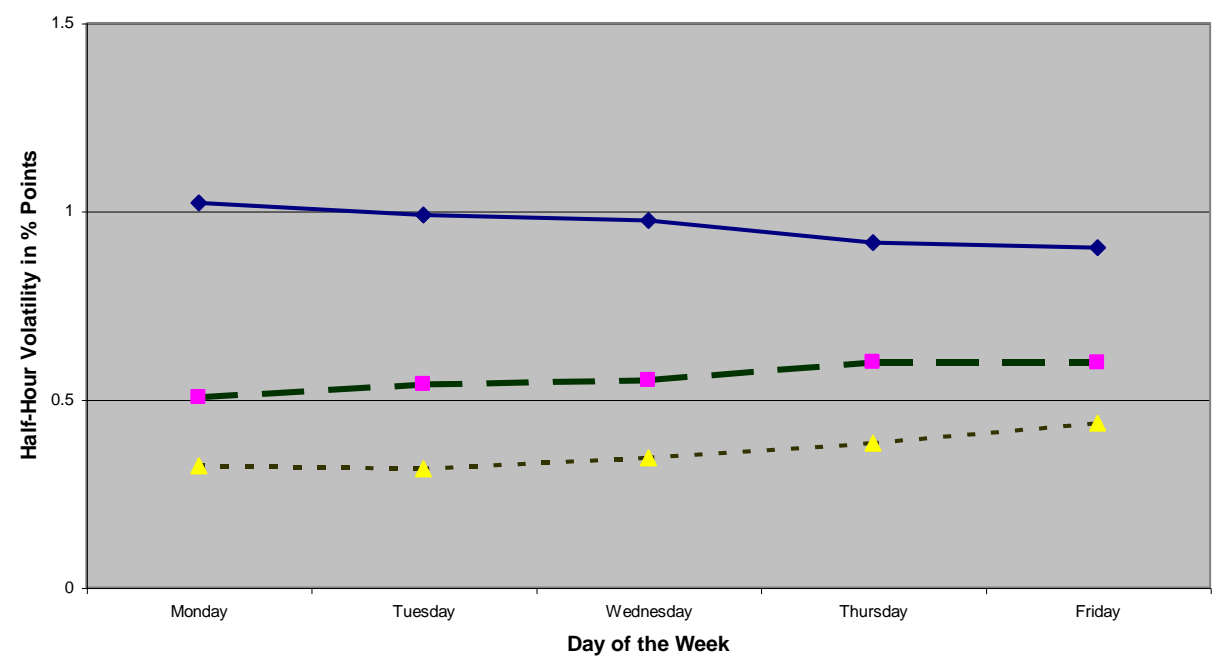

Opening half hour volatility and closing half hour volatility are calculated for each day of the week separately. The unbroken (top) line represents the opening half hour volatility. The broken (middle) line represents the closing half hour volatility for the effective 5:30 pm close, and the dotted (bottom) line represents the closing half hour volatility for the actual 8:00 pm close. Opening halfhour period does not contain the over-night price changes. The study period is June-December 2000.

Figure 5: Monday/Friday Intra-Day Volatility, New York Stock Exchange

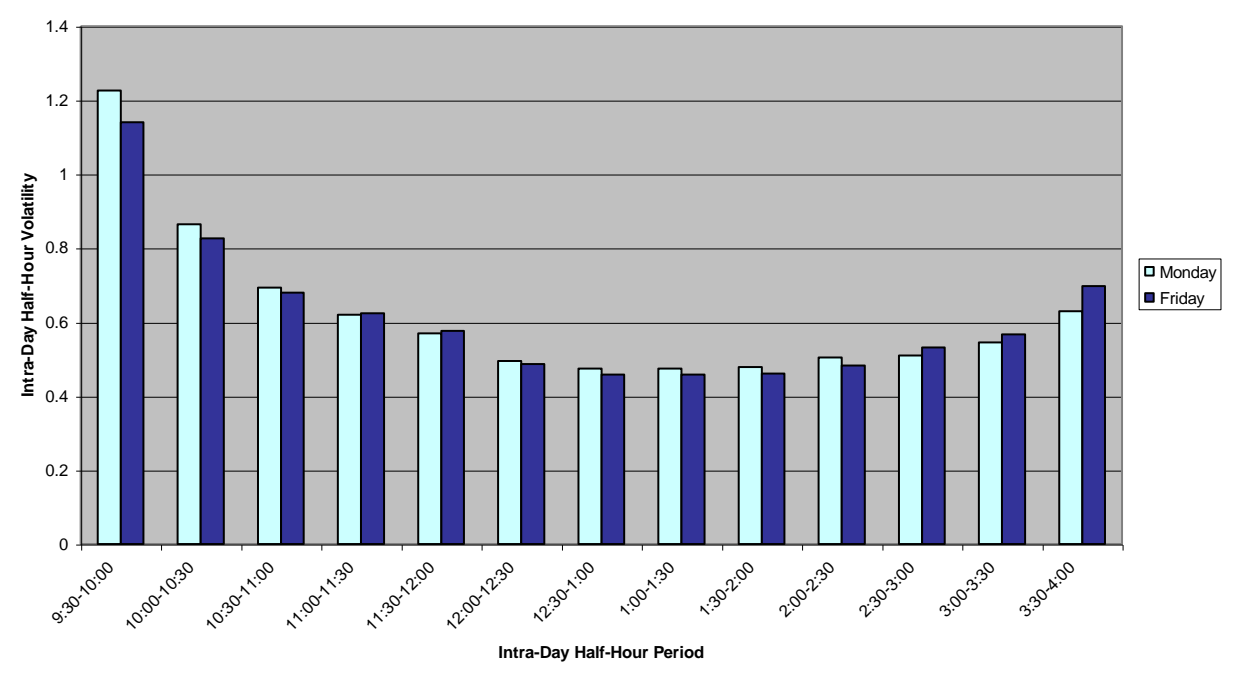

Volatility is calculated as the average standard deviation across all the stocks in the sample for each of the half hour periods during the trading day. The lighter colored bars denote the Monday intra-day volatilities and the darker colored bars denote the Friday intra-day volatilities. Opening half-hour period does not contain the over-night price changes. The study period is July-December 2000 . 
Figure 6: Monday/Friday Intra-Day Volatility, London Stock Exchange

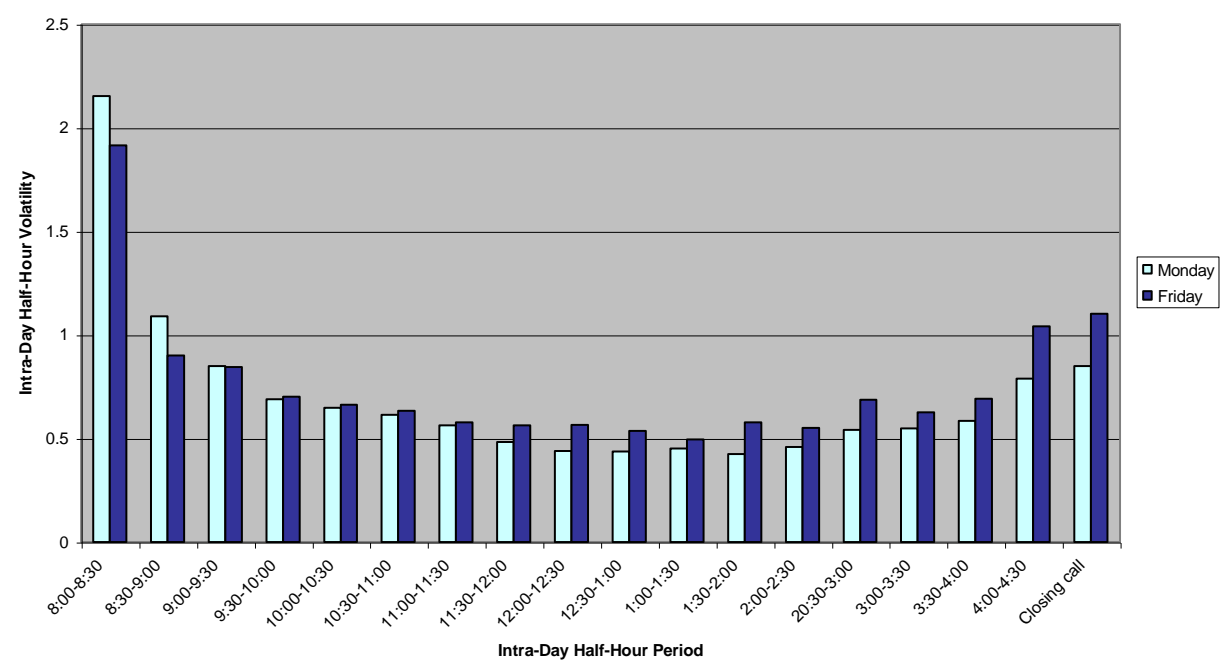

Volatility is calculated as the average standard deviation across all the stocks in the sample for each of the half hour periods during the trading day. The lighter colored bars denote the Monday intra-day volatilities and the darker colored bars denote the Friday intra-day volatilities. Opening half-hour period does not contain the over-night price changes. The study period is June-December 2000 .

Figure 7: Monday/Friday Intra-Day Volatility, Euronext Paris

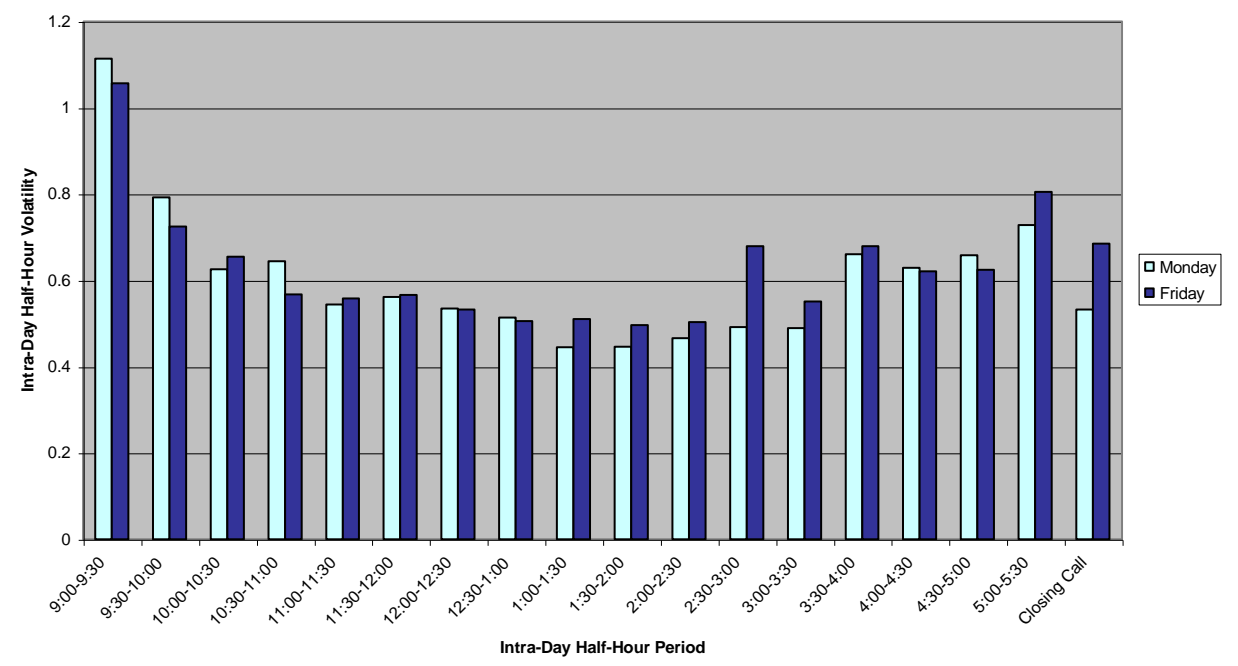

Volatility is calculated as the average standard deviation across all the stocks in the sample for each of the half hour periods during the trading day. The lighter colored bars denote the Monday intra-day volatilities and the darker colored bars denote the Friday intra-day volatilities. Opening half-hour period does not contain the over-night price changes. The study period is June-December 2000 . 
Figure 8: Monday/Friday Intra-Day Volatility, Deutsche Boerse

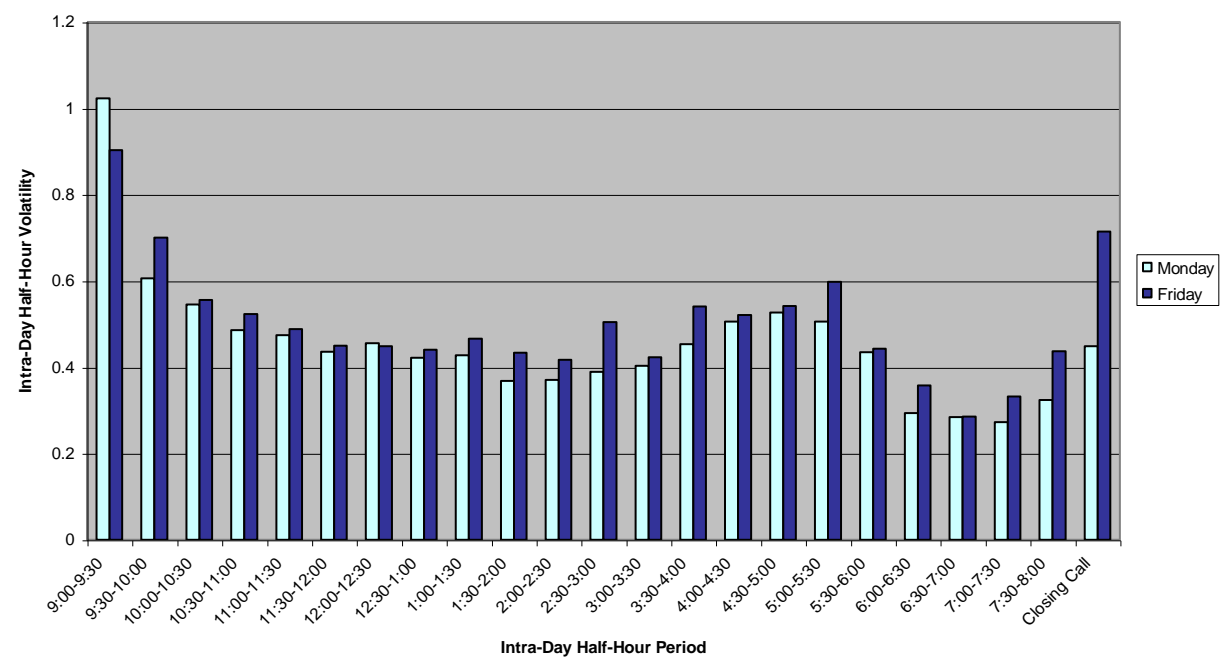

Volatility is calculated as the average standard deviation across all the stocks in the sample for each of the half hour periods during the trading day. The lighter colored bars denote the Monday intra-day volatilities and the darker colored bars denote the Friday intra-day volatilities. Opening half-hour period does not contain the over-night price changes. The study period is July-December 2000 . 
NOTES 